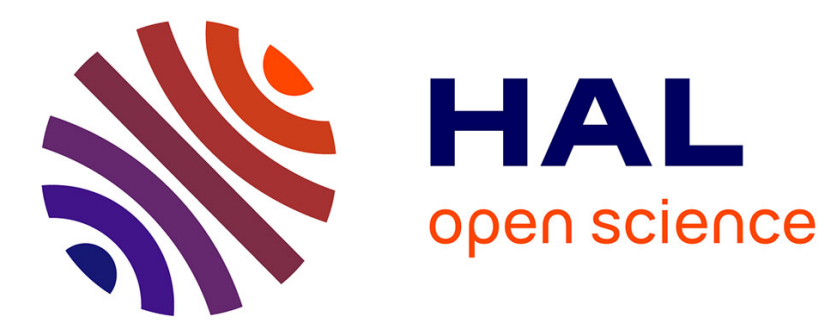

\title{
Inventaire cartographique des friches et forêts du département de l'Aube
}

Michel Cointat

\section{To cite this version:}

Michel Cointat. Inventaire cartographique des friches et forêts du département de l'Aube. Revue forestière française, 1960, 6, pp.374-381. 10.4267/2042/24285 . hal-03389695

\section{HAL Id: hal-03389695 \\ https://hal.science/hal-03389695}

Submitted on 21 Oct 2021

HAL is a multi-disciplinary open access archive for the deposit and dissemination of scientific research documents, whether they are published or not. The documents may come from teaching and research institutions in France or abroad, or from public or private research centers.
L'archive ouverte pluridisciplinaire HAL, est destinée au dépôt et à la diffusion de documents scientifiques de niveau recherche, publiés ou non, émanant des établissements d'enseignement et de recherche français ou étrangers, des laboratoires publics ou privés. 


\title{
INVENTAIRE CARTOGRAPHIQUE DES FRICHES ET FORÊTS DU DEEPARTEMENT DE L'AUBE
}

PAR

\author{
M. COINTAT \\ Ingénieur des Eaux et Forêts \\ Directeur de la S.E.F.E.
}

La dernière statistique forestière française remonte à 1912 avec la statistique générale “ Daubrée ». Il est certain que clepuis 50 ans, les peuplements se sont profondément modifiés. Les surfaces boisées ont elles-mêmes varié notablement. Si nous prenons comme exemple le département de l'Aube, l'évolution de la forêt a été la suivante, si l'on en croit les enquêtes agricoles:

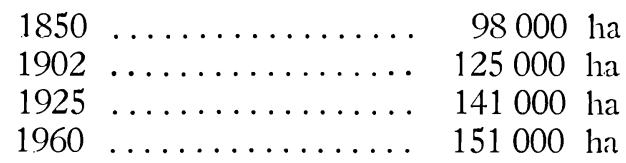

Pour la région Est de la France, l'augmentation des surfaces boisées a été dans l'ensemble depuis un siècle d'environ $30 \%$.

De nombreuses études particulières ont été consacrées ces dernières années (1) à l'inventaire cle certaines contrées ou essences, mais à l'exception du remarquable travail poursuivi en 1958-1959 par la Société le Bois Français de Papeterie sur linventaire accéléré cles ressources en bois de cellulose, il n'existe aucune étudle de synthèse : statistique forestière de production, statistique cartographique. C'est la raison pour laquelle l'Administration des Eaux et Forêts a déciclé de réaliser le recensement des peuplements forestiers de la France.

Il faut d'ailleurs reconnaitre que notre pays manque de statistiques agricoles précises. Le problème n’est pas spécial à la forêt.

(1) FABERT (Y.). - Inventaire statistique et critique des forêts lorraines. Nancy, 1956.

Noisetre (A.). - Le peuplier dans le département de l'Aube, 1957 (Congrès Internat. du Peuplier).

Corntat (M.). - Etude statistique sur les taillis-sous-futaie communaux du département de la Haute-Marne. R.F.F., octobre 1956. 
Les cartes de la végćtation de M. Gaussen ne sont pas assez précises pour l'aménagiste foncier qui travaille au stade cle la commune.

Il en est de même pour les autres travaux qui ont été poursuivis clans ce clomaine. La carte des systèmes agricoles de la France par M. Vandamme, basée sur la statistique de 1937 par canton, ne clistingue ni les forêts, ni les landes, ni les étangs. La carte d'ćvolution des tcroirs français au $1 / 400000^{\mathrm{e}}$ de M. Perpillou est intéressante pour montrer l'historique des cultures, mais a été établie d'après le cadastre de 1913. La carte de l'utilisation des terres cn France par M. J. Klatzmann ne reproduit que des pourcentages cle culture d'après l'enquête de 1942.

L'Atlas des structures agraires de la France au $1 / 200000^{\mathrm{e}}$ qui est en cours d'établissement sous la direction de M. A. Cirolley à partir des photos aériennes, répond déjà beaucoup mieux aux désirs de l'Agronome et de l'Aménagiste.

Sans atteindre la précision scientifique d'une cartographie parcellaire de la végétation, telle que la conçoit M. Kunnholtz-Lordat, il apparait souhaitable de créer un outil de travail, un document agricole et forestier, à l'échelle du plan directeur, pouvant permettre de définir dans chaque commune une politique agricole d'action.

Dans nos départements de plaine des provinces de Champagne, Lorraine, Bourgogne et Franche-Conté, à taillis-sous-futaie de chêne, hêtre et charme, nous connaissons particulièrement mal les surfaces en futaie résineuse, provenant de plantations souvent récente's, et les surfaces plantées en peupliers, essence qui n'était pas comprise clans la statistique Daubrée.

Nous avons donc pensé, dans le carlre de la mission de la Société d'Etucle des Friches et Taillis Pauvres de l'Est (S.E.F.E.), société l'économie-mixte créée en 1958, en accord avec le Ministère de l'Agriculture et le Commissariat Général au Plan et présiclée par M. le Sénateur Pisani, qu’il serait intéressant d'établir suivant une première analyse, un inventaire cartographique simplifié des forêts des départements de l'Est.

La S.E.F.E. a été chargée suivant le programme prévu au III $^{\mathrm{e}}$ plan d'équipement et de modernisation, de réaliser l'inventaire des terres incultes et il était facile en même temps que ce travail de reporter sur des plans au $1 / 20000^{\mathrm{e}}$ les principaux types de forêt.

Depuis environ un an, 1500 communes ont été inventoriées. Le clépartement de l'Aube a été le clépartement pilote et notre propos est de donner dans cette brève étude les résultats généraux de ce premier inventaire. 
Tout d'abord, comment a-t-on procédé?

La représentation cartographique a été la suivante:

Vert: Forêts feuillues, pratiquement taillis et taillis-sous-futaie.

Jaune: forêts résineuses, principalement plantations de pins sylvestres et de pins noirs.

Jaune strié cle rouge: peuplements résineux rabougris sans grande valeur économique (notamment Champagne crayeuse).

Bleu: peupleraies (les peupliers isolés cnt été indiqués par des points bleus) (un point représentant 5 arbres).

Rouge: friches, landes, terres incultes, marais, tourbières, rochers, etc...

Chaque type de friches: friches rases, genevriers, 1)roussailles, accrus feuillus, pins épars, friches marécageuses, a été précisé par cles surcharges noires conventionnelles: hachures, pointillés, tirets, croix, ronds, quadrillés, etc...

Blanc: territoire cultivé.

Munis de plans communaux au $1 / 20000^{\mathrm{e}}$, les enquêteurs relèvent tout d'abord sur le terrain la cartographie des friches et des forêts, suivant une technique maintenant au point revenant à $0,030 \mathrm{NF}$ par hectare en moyenne, scit environ une journée par territoire communal.

Cette technique - ou mieux cette pratique d'inventaire comprend en premier lieu une cléfinition aussi exacte que possible cles types de friches. Il était nécessaire de se mettre bien clans l'oxil les clifférentes formations colonisant les terrains abandonnés, et qu'il n'est pas toujours facile de distinguer entre elles: limites souvent imprécises entre les broussailles et les accrus, entre les accrus et le taillis proprement dit, etc...

Il a été convenu que clans le cloute, le type le plus évolué aurait la pricrité. Exemple: une friche à genevriers et pins clisséminés est portée sur les plans comme friche à résineux épars.

Dans certaines régions, une autre difficulté survient lorsque l'agriculteur entoure un terrain cle parcours d'une clôture sommaire, pour y faire pâturer quelques bêtes. On considère qu'il y a friche. lorsqu'il n'y a pas eu de, pratique culturale depuis longtemps (ni fauche, ni retournement, ni engrais) et lorsque 40 à $50 \%$ de la surface sont occpés par une végétation inalibile.

Pour éviter les erreurs d'interprétation, un agent technique est chargé cle superviser les enquêteurs afin d'harmoniser les relevés, en vérifiant le travail par sondages.

En outre, les prospections sur le terrain, réclament une pratique qui dépend de chaque enquêteur, et qu’il est difficile de préciser: soleil cle côté pour mieux apprécier la végétation, versants examinés cles crêtes et non cles fonds de vallons, pour juger avec certitude de la densité des parties plus ou moins boisées, etc ... 


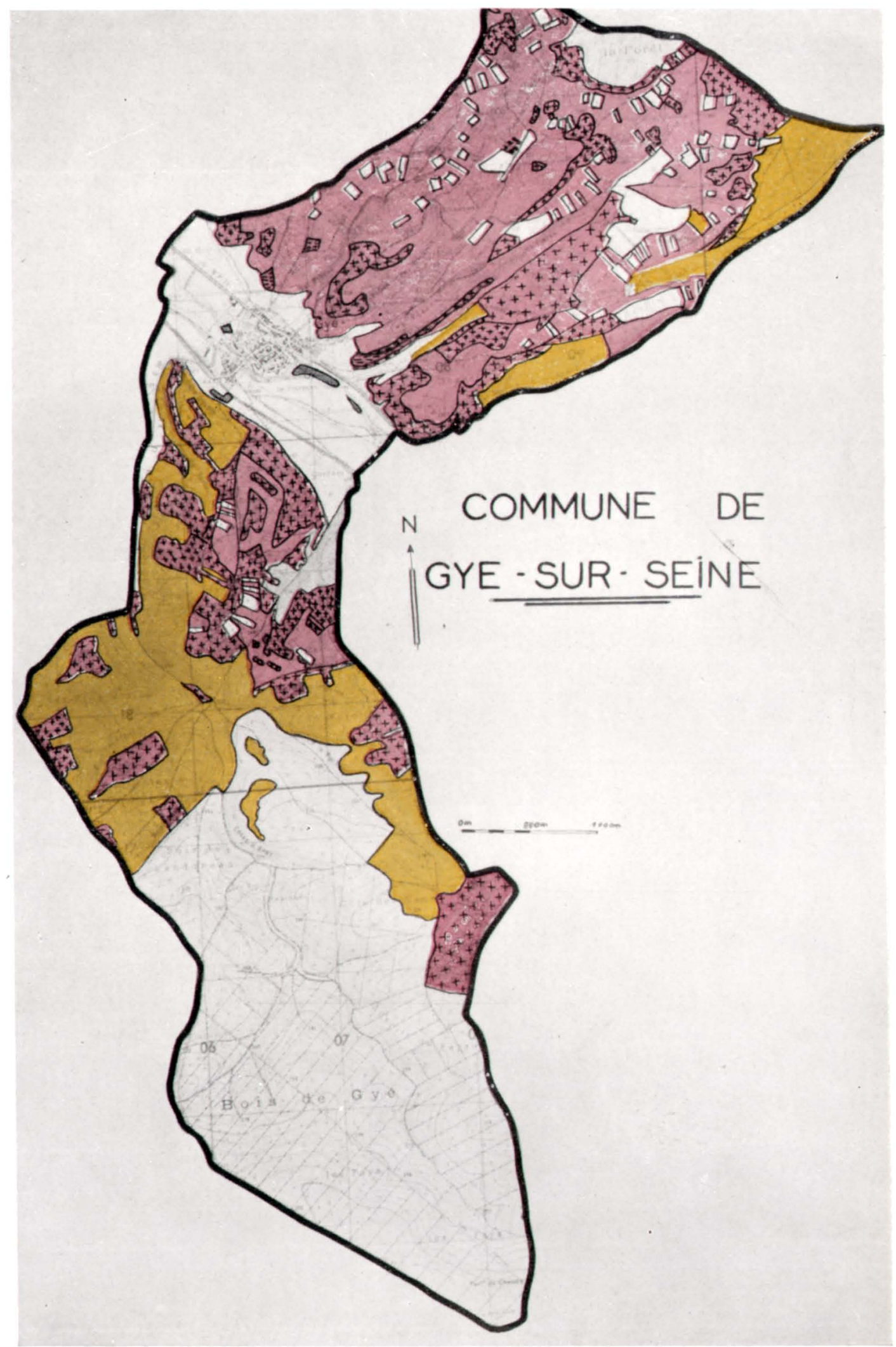


En même temps, une fiche de renseignements est établie par commune: relief, géologie, hydrographie, essences forestières, utilisation ancienne des friches, etc... suivant une méthode élaborée par le service forestier haut-marnais, lors d'une enquête forestière départementale réalisée en 1952.

Les plans brouillons transmis au bureau de dessin sont vérifiés et corrigés à l'aide de photos aériennes. Il n'est pas possible d'utiliser, sans risques d'erreurs importantes, directement les photos aériennes, par contre, la vérification des enquêtes sur le terrain par les photographies est simple et surtout indispensable pour préciser les contours.

Les surfaces des différentes végétations sont calculées suivant l'importance des lots, soit au planimètre, soit à la grille planimétrique. Les sondages effectués l'année dernière et les études parcellaires réalisées dans certaines communes de l'Aube ont montré que la précision de ces enquêtes était de l'ordre de 7 à $8 \%$.

Dans un troisième stade, les plans I.G.N. au $1 / 20000^{\circ}$ sont découpés et collés sur du papier Canson et les couleurs sont reportées.

Le plan original est enfin adressé à la photographie et reproduit en photo couleur clans le format unique $18 \times 24$ pour permettre le classement et la reliure.

Il a été adopté de classer les communes (plan et fiche de renseignements) par région agricole suivant la nomenclature de l'I.N.S.E.E. et par ordre alphabétique à l'intérieur de chaque région.

Quels sont les résultats pour le département de l'Aulse? (1)

\section{Récapitulation générale}

Surface totale du département ......... 600000 ha Forêts:

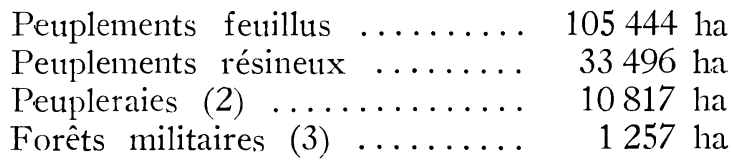

151014 ha

(1) L'inventaire des friches et forêts de l'Aube a pu être rapidement mené à bien en 1959 grâce à la bienveillance et aux encouragements de M. le Préfet et du Conseil Général de l'Aube,. de Maître Chaton, Président de la Commission des terres incultes et des chefs de service du Génie Rural, des Eaux et Forêts et des Services Agricoles. Nous leur adressons toute notre gratitude et nos remerciements.

(2) $\mathrm{Y}$ compris peupliers isolés. 200 arbres pour un hectare.

(3) Camps de Mailly et camp de Brienne. 
Friches:

Friches rases ........... 6252 ha

Friches à genevriers ......... 1335 ha

Friches à broussailles (4) ..... 6125 ha

Accrus feuillus (5) ........ 12730 ha

Résineux épars (6) ......... 16971 ha

Friches marécageuses ........ 1680 ha

Friches sur terrains militaires... 7764 ha

52857 ha

Il y a un siècle, les surfaces boisées et les surfaces incultes étaient respectivement de 98000 ha et 18000 ha. Le terroir cultivé clu département de 1'Aube a ainsi perdu près de 88000 ha et on assiste à un glissement constant de la terre vers la friche qui elle-même se transforme peu à peu et naturellement en forêt.

Les friches à genevriers qui correspondent généralement à d'anciens terrains de parcours sont peu nombreuses. Les friches à broussailles, c'est-à-dire à épines et à cornouiller, sont plus importantes et occupent l'ancien vignoble aubois. Les autres types de friches ont des origines diverses.

Les forêts sont très différentes suivant les régions agricoles, et il est intéressant cle noter cette répartition.

Les forêts feuillues se divisent en trois types principaux:

1 - les taillis-sous-futaie à hêtre et chêne clu Vignoble clu Barrois.

2 - les taillis-sous-futaie à chêne cle qualité de la Champagne Humide.

3 - et les taillis-sous-futaie à chênes petits et médiocres du pays d'Othe.

C'est en 1816 que Baltet-Petit introduisit les premiers résineux clans la Champagne Crayeuse de l'Aube, à.Echemines. Un monument commémore d'ailleurs cet événement. Les plantations de pins sylvestres sont très souvent rabougries et depuis une dizaine d'années les défrichements ont été particulièrement importants. Les chiffres ci-dessous montrent cependant que les résineux occupent encore sur la craie des surfaces appréciables.

Dans le Barrois, des plantations de pins sylvestres et quelques pins noirs ont été réalisées depuis 1860-1880. Elles s'étendent aujourd'hui naturellement dans les friches voisines.

(4) Epines noires et cornouiller sanguin principalement.

(5) Terme local pour désigner des cépées de taillis sans valeur et occupant incomplètement la forêt.

(6) Peuplements résineux rabougris (pin sylvestre) occupant moins de $50 \%$ de la surface en friche. 


\section{Répartition des forêts par région agricole (en ha)}

\begin{tabular}{|c|c|c|c|c|c|}
\hline & $\begin{array}{c}\text { Taux } \\
\text { die boisement } \\
-\end{array}$ & $\begin{array}{l}\text { Forêts } \\
\text { feuillues } \\
-\end{array}$ & $\begin{array}{c}\text { Forêts } \\
\text { résineuses } \\
-\end{array}$ & $\begin{array}{c}\text { Peu- } \\
\text { pleraies } \\
-\end{array}$ & Totaux \\
\hline Vignoble du Barrois. & $31 \%$ & 35301 & 5346 & 786 & 41433 \\
\hline Plaine de Brienne ... & $15 \%$ & $\begin{array}{l}2554 \\
355(1)\end{array}$ & $\hat{1}$ & 383 & $\begin{array}{r}2981 \\
355\end{array}$ \\
\hline Champagne Humide . & $37 \%$ & 41174 & 236 & 1056 & 42466 \\
\hline $\begin{array}{c}\text { Vallée de la Champagne } \\
\text { humide } \ldots \ldots \ldots \ldots . .\end{array}$ & $19 \%$ & 681 & 2 & 292 & 975 \\
\hline Plaine de Troyes .... & $6 \%$ & 132 & 516 & 1006 & 1654 \\
\hline Pays d'Othe ......... & $34 \%$ & 18771 & 570 & 400 & 19741 \\
\hline Champagne Crayeuse . & $19 \%$ & 4436 & $\begin{array}{l}22524 \\
\quad 902(1)\end{array}$ & 1083 & $\begin{array}{r}28043 \\
902\end{array}$ \\
\hline $\begin{array}{l}\text { Vallées de la Champa- } \\
\text { gne Crayeuse ...... }\end{array}$ & $15 \%$ & 829 & 4115 & 3628 & 8572 \\
\hline Nogentais $\ldots . . . \ldots$. & $10 \%$ & 858 & 86 & 768 & 1712 \\
\hline Vallée du Nogentais . & $25 \%$ & 748 & 17 & 1415 & 2180 \\
\hline Totaux $\ldots . .$. & $25 \%$ & 105799 & 34398 & 10817 & 151014 \\
\hline
\end{tabular}

(1) Terrains militaires.

On peut considérer que les surfaces classées dans les friches à résineux épars sont boisées à $30 \%$ environ et pour permettre une politique de production, il est nécessaire de donner la surface corrigée pour les résineux:

Boisements résineux proprement dits ..... 33496 ha

Friches à résineux épars, surface corrigée .. 5091 ha

Surface totale en résineux .........

Les peupliers sont concentrés dans les vallées. Ils constituent de magnifiques peuplements sur les bords de la Seine et de l'Aube. Cet inventaire fait apparaître une surface en peupliers plus importante que les chiffres donnés par les statistiques précédentes.

La répartition des friches est également significative suivant les régions agricoles.

Les terres incultes sont concentrées sur les plateaux calcaires Jurassiques du Barrois (anciennes vignes) et sur la craie Champenoise (savarts et triots), 20 communes (1) de l'Aube renferment plus

(1) 13 pour le Vignoble du Barrois, 2 pour la Plaine de Brienne et 5 pour la Champagne Crayeuse. 


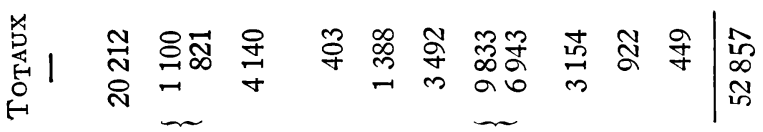

总

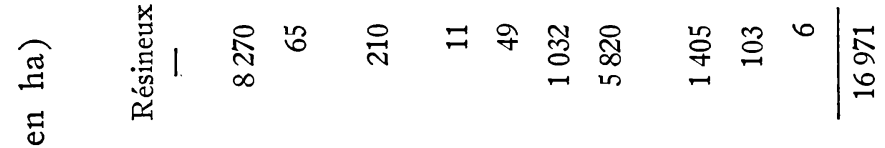

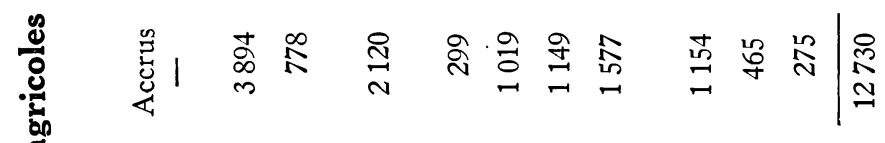

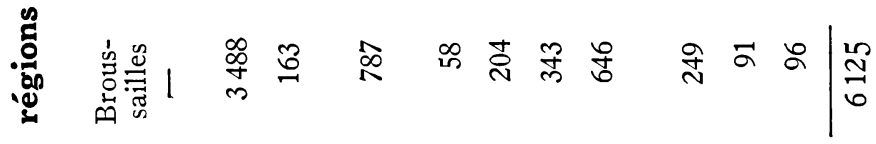

ณั

总这

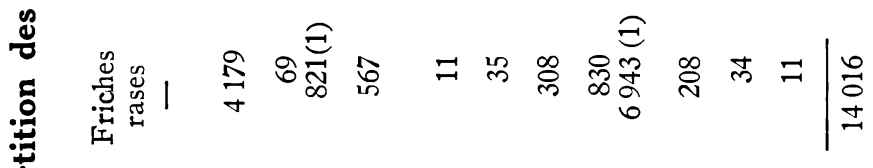

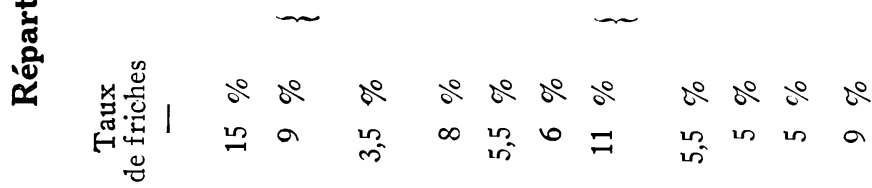

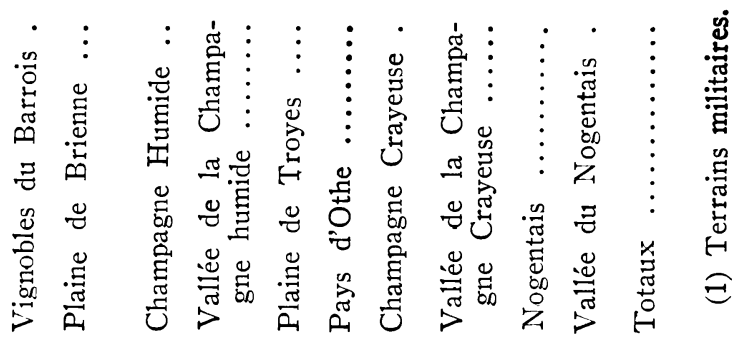


de $30 \%$ de friches. Parmi celles qui sont le plus abandonnées et où le taux de friches dépasse $50 \%$, citons: Viviers-sur-Artaut (66 \%), Neuville-sur-Seine (53\%), Poivres (70\%), Trouan-leGrand $(75 \%)$, etc...

Toutefois, l'implantation des friches clans les finages est différente suivant les contrées. Sur le plateau des Bars, les terres incultes colonisent des cantons entiers et constituent des masses importantes d'un seul tenant, nécessitant des études d'aménagement foncier précédent la remise en valeur.

Par contre, dans le pays d'Othe, où les vergers de pommiers à cidre ont été souvent abandonnés, les friches parfois nombreuses sont très disséminées et il suffirait d'un remembrement classique pour les faire disparaitre.

Les broussailles, les accrus feuillus et les résineux épars, forment la majorité des sols inutilisés du département de l'Aube. Depuis 1850, la surface des friches a triplé et l'évolution est relativement rapicle. Or, le défrichement coûte de $200 \mathrm{NF}$ à $3000 \mathrm{NF}$ l'hectare, suivant que l'on a affaire à une friche rase ou à un taillis bien ensouché. Il apparaît par conséquent urgent de promouvoir une politique de remise en valeur de ces terrains, pour la réanimation des régions telles que le plateau du Barrois. Plus on attendra, plus le céfrichement cồtera cher, la végétation arbustive accentuant rapidement son emprise d'années en années.

En résumé, le clépartement de l'Aube a servi de région pilote pour la mise au point d'un inventaire cartographique des friches et les forêts. L'échelle du $1 / 20000^{e}$ permet de fournir à l'aménagiste un document suffisamment précis et létaillé, et la Société des Friches de l'Est suivant un programme de 4 ans, cloit poursuivre ces inventaires dlans 12 départements de l'Est.

La cartographie de la Meuse et de la Haute-Marne sera terminée au printemps 1960. Les résultats de l'inventaire seront également publiés avant la fin de l'année 1960 pour les départements de la Côte-d’Or, de la Haute-Saône et de la Marne.

Le 20 avril 1960. 